\title{
Algumas Questões sobre o Desenvolvimento da Epidemiologia na América Latina
}

\author{
Rita Barradas Barata ${ }^{1}$ \\ Maurício Lima Barreto ${ }^{2}$
}

\begin{abstract}
Resumo: Este artigo apresenta de maneira panorâmica algumas questões referentes ao estado atual do conhecimento e da prática epidemiológica no Brasil, relacionando tais desenvolvimentos com o movimento internacional no campo. Inicialmente é apresentado, de maneira sucinta, o desenvolvimento da área na última década. Em seguida, caracteriza-se a crise paradigmática da disciplina e apontam-se algumas questões teóricas e metodológicas relevantes no sentido da definição de um novo paradigma. Na seqüência, são abordadas questōes relativas à incorporação dos conhecimentos epidemiológicos aos serviços de saúde apontando alguns dos desafios apresentados e, finalmente, considera-se as tendências de desenvolvimento da corrente hegemônica e também contrahegemônica da epidemiologia.

Palavras-Chave: Epidemiologia; Tendências; América Latina.

Summary: This paper presents in a panoramic way some relevant questions related to the present status of the epidemiologic knowledge and practices in Brazil and its relationship with the international movement in this field. Following, its paradigmatic crisis was characterized and some relevant theoretical and methodological questions towards the definition of a new paradigm were raised. Other topic discussed was about the utilization of the epidemiological knowledge in the health services. At the end, the trends in the development of the hegemonic epidemiology were considered, as well as its contra-hegemonic counter-part.
\end{abstract}

Keywords: Epidemiology; Trends; Latin America.

\footnotetext{
Departamento de Medicina Social - Faculdade de Ciências Médicas, Santa Casa, São Paulo.

Instituto de Saúde Coletiva - UFBa, Salvador, Bahia.
} 


\section{Introdução}

A epidemiologia tem apresentado, em nível mundial, um intenso e rico desenvolvimento no decorrer deste século, principalmente, nas últimas décadas. Os conhecimentos gerados pelos estudos epidemiológicos têm interferido no cotidiano das pessoas à medida que sua divulgação tem implicado mudanças de hábitos e comportamentos. Entre os muitos exemplos poderíamos citar a questão dos hábitos alimentares e das atividades físicas e a sua associação com as doenças cardiovasculares. A presença crescente dos produtos da pesquisa epidemiológica no cotidiano das pessoas e a ampliaçào de seu campo de aplicações, que extrapola do processo saúde-doença para as questōes relativas à produção e oferta de serviços de saúde, entretanto, não são capazes de ocultar os problemas existentes na disciplina. De um lado, questões relativas ao âmbito interno, de ordem teórica e metodológica, e de outro, questões relativas à utilização do conhecimento produzido no sentido de solucionar problemas de saúde.

Este artigo se propõe a apresentar um elenco desses problemas e discutir algumas das tendências presentes no campo, com ênfase nos enfoques desenvolvidos no Brasil e na América Latina, sem perder de vista os vínculos mais gerais entre essas produções e aquelas feitas nos países de primeiro mundo.

\section{A Epidemiologia no Brasil}

No decorrer dos últimos dez anos, a Epidemiologia no Brasil apresentou ritmo acelerado de crescimento verificado através do aumento da produção científica, do aumento do número de seus praticantes, da demanda por contribuições da epidemiologia na reorganização dos serviços de saúde, e, também, do desenvolvimento e amadurecimento do debate conceitual e metodológico. Este crescimen- to esteve ancorado no aumento dos cursos de pós-graduação senso estrito e senso lato, no apoio institucional representado pela Organização Panamericana de Saúde, pela criação do Centro Nacional de Epidemiologia da Fundação Nacional de Saúde e no trabalho da comissão de Epidemiologia da ABRASCO.

Apesar da inexistência de um diagnóstico atualizado da produção recente na área, avalia-se através de indicadores tais como a proporção de artigos publicados nas revistas de Saúde Coletiva e de temas livres apresentados nos congressos de Epidemiologia e Saúde Coletiva, e de livros e capítulos de livros publicados, que a produção científica cresceu significantemente, nos últimos anos (ABRASCO, 1990; ABRASCO, 1992; ABRASCO, 1995; Pellegrini et al., 1995).

A própria posição ocupada pela investigação científica no conjunto de preocupações da área sofreu alteração importante nesse período. Se recordarmos a primeira Reunião Nacional sobre Ensino e Pesquisa em Epidemiologia, promovida pela ABRASCO, em Friburgo, em 1984 (ABRASCO, 1986), chamará nossa atenção a importância concedida ao ensino e à formação de residentes, ficando as questōes relativas à pós-graduação senso estrito e à pesquisa científica em segundo plano.

Após a criação da Comissão de Epidemiologia, ainda em Friburgo, vários seminários e reuniões se sucederam dando origem ao "Ciclo de Itaparica", que culminou na elaboração do I Plano Diretor para o Desenvolvimento da Epidemiologia no Brasil (ABRASCO, 1989). Neste documento, que orientou os trabalhos da comissão entre 1990 e 1994, as tarefas foram agrupadas em três blocos: ensino, pesquisa e práticas em serviços, sendo que as duas primeiras apresentaram maior densidade de propostas.

Outro marco na história do desenvolvimento da Epidemiologia brasileira foi a realização do I Congresso Brasileiro de Epidemiologia, em Campinas, em 1990. A despeito da crise vigente no país, o evento teve larga 
adesão, tendo o número de participantes nacionais e estrangeiros superado as expectativas dos organizadores. Além das dimensões, a qualidade dos trabalhos trouxe grande impulso às atividades da comissão. Este novo patamar do movimento da epidemiologia brasileira teve continuidade no II Congresso, realizado em Belo Horizonte, em 1992, e no III Congresso, em Salvador, em 1995.

A partir da criação do CENEPI pela Fundação Nacional de Saúde, a participação de docentes e pesquisadores das Universidades e Institutos de pesquisa na capacitação de profissionais de saúde e no encaminhamento de questôes relativas às práticas em serviço tornaram-se freqüentes, dando origem a uma colaboração efetiva e proveitosa entre universidade e serviços de saúde. Tal envolvimento pode ser constatado na elaboração do II Plano Diretor para o Desenvolvimento da Epidemiologia no Brasil, para o período de 1995 a 1999, no qual o destaque para as questões referentes às práticas em serviços de saúde é bastante grande, equiparandose às questões relativas ao ensino e à pesquisa (ABRASCO, 1995).

A expressiva participação de pesquisadores estrangeiros, provenientes da Península Ibérica, países nórdicos, Estados Unidos, Canadá e América Latina, durante o III Congresso Brasileiro de Epidemiologia, ocasião em que também se realizaram o II Congresso Ibero-americano e o I Congresso Latino-americano de Epidemiologia, apontam um novo momento de inflexão no desenvolvimento da epidemiologia brasileira, assinalando a importância que a colaboração com outros grupos de pesquisadores possa ter no amadurecimento da área.

Frente aos avanços já alcançados e aos desafios ainda por vencer, a Comissão de Epidemiologia assumiu o compromisso de dar continuidade à organização de congressos, seminários e reuniões técnicas e abrir uma nova frente de trabalho com o lançamento da Revista de lipidemiologia, da ABRASCO, conso- lidando assim o crescimento verificado na produção científica e tecnológica da disciplina.

Todo esse crescimento ocorre em meio a uma "crise de identidade" da epidemiologia enquanto disciplina científica, crise esta identificada não apenas por pesquisadores do Terceiro Mundo, como ocorreu durante as décadas de 70 e 80 , mas também polemizada e debatida por pesquisadores dos países desenvolvidos. Esta crise e as questões que ela levanta para os praticantes da investigação científica em Epidemiologia serão o objeto de nossa discussão.

\section{A Crise da Epidemiologia Hegemônica e as Críticas a Ela Formuladas}

Partindo dos critérios propostos por Thomas Kuhn (1989) e, agregando a eles novos elementos, podemos identificar a crise hoje existente na produção da ciência epidemiológica manifesta através de insuficiências, paradoxos, anomalias e limites (Almeida-Filho, 1994). Dentre as insuficiências podemos mencionar a incapacidade de formular um conceito de saúde positivo e a dificuldade em operacionalizar sua própria concepção de doença no plano coletivo, tornando-se assim dependente das concepções elaboradas pela Clínica para o plano individual. Os paradoxos podem ser observados cotidianamente a partir da divulgação, na imprensa leiga e especializada, de resultados de pesquisas que trabalham com a mensuração de riscos e o estabelecimento de associações entre tais medidas e os chamados fatores de risco, o que demonstra o grau de discordância e confusão prevalecente no campo (Taubes, 1995). A inadequação entre teorias, métodos e práticas gera insatisfação e inquietação entre os pesquisadores menos propensos a conformar-se às práticas da "ciência normal" (Czeresnia, 1993).

Face a esses impasses as ciências empíricas têm optado pelo maior aprimora- 
mento técnico, aumentando o grau de positividade de seus enunciados e restringindo as condições de validade destes. Deste modo, as tentativas de superação da crise restringemse aos aspectos da "teoria em si", isto é, ao sistema lógico-conceitual, sem considerar a possibilidade de desenvolvimento de uma teoria que obedeça aos interesses emancipatórios e enriquecedores, e, portanto, aos compromissos éticos da investigação científica, na direção de uma prática realmente emancipadora (Mendes-Gonçalves, 1990; Ayres, 1994).

\section{Questões Relativas ao Desenvolvimento Interno da Disciplina}

O principal problema teórico da Epidemiologia é reconhecer-se e reafirmar-se como teoria. Seu objeto real é a "gênese e o devir dos processos saúde-doença em populações concretas" (Mendes-Gonçalves,1990), colocando para a disciplina o desafio de compreendêlos no "seio de uma sociedade complexa, plural e contraditória, atravessada por práticas institucionais e constituída por agentes históricos concretos" (Almeida-Filho, 1995).

Há dois pressupostos básicos para orientar a discussão sobre o objeto científico: o primeiro afirma a precedência do objeto real sobre o objeto do conhecimento, isto é, define as necessidades concretas dos homens como geradoras de teorias; o segundo afirma a precedência do objeto real sobre o método, isto é, a definição da disciplina científica e sua individualização no conjunto de abordagens possíveis é dada pelo objeto de estudo e não pelos métodos empregados para estudálo (Mendes-Gonçalves, 1990).

O objeto, como lembra Almeida-Filho (1994), é diferente da representação da coisa, não é o equivalente abstrato da coisa real. $O$ objeto construído na e para a investigação científica guarda uma relação de referência para com o objeto concreto. O desafio está em superar as limitações representadas pelo caráter instrumental, materializado no conceito de risco, na busca de objetos-modelo com capacidade heurística, sem entretanto romper, descaracterizando, os limites da disciplina. Há diversas tentativas, no campo, de elaboração de objetos mais complexos e totalizados tais como o perfil epidemiológico de Breilh, o nexo biopsíquico de Cristina Laurell, o modo de vida de Cristina Possas e a teoria da saúde, com o conceito de reprodução social de Juan Samaja (Almeida-Filho, 1992).

Em sentido contrário aos esforços destacados no parágrafo anterior, existem movimentos no sentido de uma maior formalizaçào que, se por um lado, representam tentativas de ampliação dos objetos sobre os quais um hipotético "método epidemiológico" viria a ser aplicado; por outro, acabam por determinar uma redução na identidade científica da disciplina ao descaracterizar a especificidade de seu objeto (Ayres, 1994).

A segunda questão refere-se aos pressupostos lógicos da epidemiologia enquanto disciplina científica. Vários autores têm apontado as limitações que a adoção da lógica formal tem significado para a possibilidade de superação da crise na disciplina. Um dos aspectos que tem sido objeto de reflexão na epidemiologia diz respeito ao núcleo mesmo da lógica formal, isto é, ao princípio da identidade, com seus corolários da não-contradição e da obrigatoriedade de toda proposição possuidora de sentido ser identificada como verdadeira ou falsa em termos absolutos (Castiel, 1995). Outros aspectos lógicos relevantes são as relaçōes de externalidade entre o processo saúde-doença (parte) e a sociedade (todo), os procedimentos inferenciais centrados exclusivamente nos processos indutivos ou dedutivos, recusando outras formas de inferência, o predomínio do determinismo causal e a recusa de outras formas de determinação, a redução do real a um agregado de indivíduos e a negação dos vínculos relacionais constitutivos da totalidade (CORISCO, 1993). 
Além das questões relativas ao sistema de lógica em si há um número relativamente grande de oposições ou antinomias presentes na discussão, dentre as quais cabe destacar a oposição entre teoria e prática, sujeito e objeto, individual e coletivo, biológico e social etc. (Almeida-Filho, 1994). Outro aspecto freqüentemente posto em questão é o relativo à construção de proposições verdadeiras (Ayres, 1994; Czeresnia, 1993). A teoria crítica se impõe como tarefa a busca de soluções, em outro sistema de lógica, para cada um desses problemas.

O terceiro conjunto de questões refere-se aos métodos de investigação, caracterizados por um certo "cientismo", ou seja, impessoalidade dos métodos, rigor técnico, objetividade, abordagem unidimensional de sujeitos privados, para os quais as variaçôes quantitativas de qualidades homogêneas serão objeto de investigação e biologicismo, que resulta em normalização metodológica e elaboração de um "discurso pobre e deslocado da vida" (Castiel, 1995; Ayres, 1992; Czeresnia, 1993). A fragmentação e a especialização crescentes, sob o primado do conceito de risco, e a falsa oposição entre observação e experimento, métodos descritivos e analíticos representam obstáculos à busca de alternativas metodológicas que permitam a compreensão do processo saúde-doença enquanto sistema complexo de determinações (Almeida-Filho, 1994 \& 1995).

As saídas apontadas para essas insuficiências vão na direção dos estudos de complexidade, superação do indutivismo, revisão da noção de experimentação, crítica ao pretenso objetivismo das evidências experimentais, reconhecimento dos procedimentos de implicação presentes na construção de evidências, assim como de outros procedimentos de negociação, aprimoramento dos estudos de agregados e revisão crítica da "falácia ecológica", buscando a pluralidacle dos métodos, a utilização de analogias e metáforas como ferramentas de criação/invenção, a elaboração de "famílias de modelos" e a constru- ção do coletivo como unidade de observação, análise, interpretação e intervenção (Almeida-Filho, 1995; Castiel, 1995; CORISCO, 1993; Wing, 1993; Samaja, 1992; Amann \& Knorr-Cetina, 1990; Morgenstern, 1982).

O quarto grupo de questões é relativo aos problemas técnicos derivados das opções teóricas e metodológicas dominantes. Como conseqüência das escolhas feitas nos planos teórico, lógico e metodológico as técnicas de quantificação matemática são consideradas as únicas capazes de contribuir na formulaçào de proposições verdadeiras, objetivas e neutras sobre as associações entre variáveis isoladas e delimitadas e a ocorrência de doença (risco) em grupos de indivíduos recortados segundo sua condição de exposição. Entretanto, esta escolha não está livre de problemas e limitações na medida em que não é possivel garantir a comparabilidade entre expostos e não expostos (nos termos esperados pela receita da experimentação), nem equacionar de maneira satisfatória a categoria exposição. Há diversas tentativas de sofisticação dos modelos matemáticos visando superar as limitações das funções lineares univariadas e multivariadas, bem como propostas para análises em múltiplos níveis, que incorporam variáveis do nível individual, familiar, de classes etc. O maior desafio está justamente na reconstrução dos nexos existentes no sistema complexo entre o processo saúde-doença, por um lado, e os indivíduos e a sociedade, por outro. Necessariamente, o uso de técnicas exclusivamente quantitativas significa uma restrição injustificada face aos problemas apontados. Uma vez que os procedimentos técnicos analíticos rompem esses vínculos e fragmentam a realidade, a interpretação adequada, isto é, mais próxima do real, deverá reconstruir, na medida do possível, essas relações sob pena de não alcançar efetividade (Wing, 1993b; Von Korff et al., 1992).

Em decorrência dos problemas já apontados coloca-se para a epidemiologia o desafio 
da superação dos limites disciplinares na apreensão do processo saúde-doença em sua dimensão coletiva. Trata-se da questão da transdisciplinariedade exigida pela própria complexidade do objeto de investigação: "troca e diálogo" entre diferentes saberes científicos, entre saberes científicos e saberes do cotidiano, entre discursos e práticas. É necessário apostar na pluralidade dos métodos na construção de "teorias verdadeiras", onde a positividade decorra de um procedimento compartilhado intersubjetivamente, a normatividade seja a expressão da capacidade de implementação "práxica" de seus produtos e cuja autenticidade repouse na efetiva comunicação. Teoria verdadeira, no sentido ético de verdade, por implicar uma práxis emancipadora, não se limitando ao campo de ação da razão instrumental (Almeida-Filho, 1995; Ayres, 1994; CORISCO, 1993; Mendes-Gonçalves, 1990; Samaja, 1992).

\section{Questões Relativas às Práticas Epidemiológicas em Serviços de Saúde}

As ações de saúde voltadas para o controle das doenças transmissiveis e das epidemias são muito antigas e constituem as práticas mais tradicionais no campo da Saúde Coletiva, ao lado das ações de saneamento ambiental.

A necessidade de reorientar e reorganizar os serviços de saúde, de forma a atender aos princípios de universalização, eqüidade, integralidade, democratização, controle social e qualidade na prestação dos serviços, tem levado os profissionais de saúde a procurar, no campo disciplinar da epidemiologia, respostas para as várias questões formuladas por esse processo de mudanças. Nesse movimento de revisão, com vistas à construção do Sistema Único de Saúde (SUS), os epidemiologistas inicialmente procuraram afirmar que as contribuições da disciplina não deveriam ficar restritas às tradicionais atividades de vigilância epidemiológica e que a racionalidade epidemiológica encerra possibilidades mais amplas de atuação, não se restringindo ao fornecimento de elementos técnico-científicos para um conjunto limitado de problemas de saúde (Barata, 1993).

Segundo Paim (1992), a Saúde Coletiva é um campo de práticas cujo objeto é constituído pelas necessidades sociais de saúde, sendo as condições sanitárias a expressão das condições materiais de existência dos grupos sociais. No sistema capitalista a estrutura de necessidades necessárias ${ }^{3}$ sofre um processo de alienação, reduzindo-se à dimensão de consumo individual. $\mathrm{Na}$ esfera da saúde, as necessidades sociais se vêem restritas àquelas que estão vinculadas à preservação e reparação da força de trabalho e àquelas que correspondem à realização da necessidade político-ideológica expressa nos "direitos dos consumidores". O que se pretende pontuar aqui é que as necessidades humanas sofrem um processo de empobrecimento, no sentido de que os indivíduos são reduzidos ao papel de meros consumidores de serviços assistenciais, dietas especiais, exercícios, alimentos, vitaminas etc. (Mendes-Gonçalves, s/d).

Nessa lógica do mercado em que o importante é acumular e consumir, a organização dos serviços de saúde é fortemente marcada pela ótica administrativa, isto é, pela busca da maior adequação entre meios e fins, tomando como momento privilegiado a organização e disposição dos meios (Paim, 1992). A existência de um problema de saúde por si só não basta para caracterizá-lo como necessidade social de saúde. Tal caracterização depende de uma "leitura" efetuada pelo Estado a partir de

s Em português é inevitável a redundância entre necessidade como impulso, apetite, desejo e necessidade como dever ser, vínculo obrigatório. Em outras línguas temos duas palavras tais como need e necessity em inglês, besoin e necessité em francês e bedürfnis e notwendigkeit em alemão. 
uma análise das repercussões econômicas, das pressōes políticas e das percepções sociais relativas ao problema. Essa "leitura" depende, portanto, e fundamentalmente, da teoria acerca das relaçôes Estado-sociedade e da possibilidade de expressão democrática das diferentes estruturas de necessidades dos grupos sociais (Paim, 1992; Barata, 1990).

Os sistemas locais de saúde aparecem como recurso tático no processo de transformação e redirecionamento das políticas de saúde, no interior de propostas de modernização do Estado e racionalização dos recursos financeiros, materiais e humanos na prestação dos serviços de saúde. Determinados atores sociais, profissionais de saúde, partidos políticos e movimentos populares organizados, buscam nesse processo formas de maior democratização do Estado e de construção de mecanismos de solidariedade social que possam facilitar a superação das desigualdades, pelo menos no nível do consumo de bens e serviços (Barata, 1990).

A epidemiologia, enquanto disciplina científica, se constitui tomando como objeto de conhecimento e de práticas o processo saúde-doença no plano coletivo, supraindividual. Nesta dimensão, os corpos não podem ser tomados em suas características exclusivamente biológicas, mas, necessariamente, deverão ser tratados como corpos sociais. Para permitir a previsão de fenômenos e a proposição de medidas de intervenção, a solução encontrada pela epidemiologia foi a redução do social, isto é, das complexas determinações sociais, a um conjunto de atributos passíveis de objetivação através de técnicas e instrumentos de quantificação. Simultaneamente, procedeu-se à "naturalização" do social, esvaziando os processos sociais de seu conteúdo político intrínseco, transformando-os em "legalidades", menos ou mais imutáveis, postas à margem da história. Desta forma, a epidemiologia pode se tornar um instrumento racionalizador para as práticas de saúde em sua dimensão coletiva (Barata, 1990; Barreto \& Alves, 1994).
Entretanto, partindo das necessidades sociais de saúde segundo uma perspectiva não alienada, isto é, buscando tomar as necessidades como expressão das potencialidades humanas integrais, a epidemiologia procura inverter o pólo do planejamento em saúde, retirando o foco das práticas de saúde organizadas nos serviços. Esta inversão do pólo de atenção deve permitir a apreensão do conjunto de necessidades tais como elas se apresentam na sociedade, na população em seu território, e não da forma como são recortadas, redefinidas e priorizadas pelos serviços de saúde.

A captação das necessidades de saúde, ou seja, sua objetivação constitui o diagnóstico epidemiológico de saúde. Este diagnóstico representa o objeto construído para orientar a organização das atividades de intervenção. Ele representa a "demanda potencial", isto é, o conjunto dos problemas socialmente postos e que carecem de soluções ou respostas sociais (Castellanos, 1990). Partindo do princípio de que os serviços de saúde deveriam organizarse tendo por finalidade a satisfação das necessidades sociais de saúde, tal como elas se apresentam nos planos coletivo e individual, o diagnóstico epidemiológico deverá orientar os planos de ação, delimitando o campo de atuação e as opçôes possíveis (Barata, 1990).

Além de fornecer uma "nova lógica" para a reorientação dos serviços de saúde a epidemiologia também deverá ter papel destacado na avaliação desses serviços. A avaliação das ativiclades, programas e ações para além de seus aspectos técnicos, que envolvem a verificação de impacto e aspectos relativos aos custos financeiros, deverá levar em conta a questão do atendimento das expectativas depositadas pelos grupos sociais na eficácia das intervenções. Coloca-se mais uma vez aqui a questão da democratização dos serviços e da possibilidade de controle social, na medida em que a avaliação deixe de levar em conta apenas os aspectos internos à organização das práticas para avaliar as transformações realmente operadas nas condições de vida dos grupos sociais. 
Nesse envolvimento da epidemiologia com o mundo dos serviços de saúde e das práticas de prevenção e controle das doenças, freqüentemente emergem questôes relativas à validade ou utilidade dos conhecimentos epidemiológicos, quer seja no âmbito mais restrito dos indivíduos, quer seja no âmbito mais ampliado da sociedade. Até que ponto a coleção interminável de fatores de risco identificados nas pesquisas epidemiológicas podem ser aplicados como elementos diagnósticos ou prognósticos para os indivíduos? Em que medida os riscos atribuíveis calculados em pesquisas epidemiológicas podem ser observados na operacionalização de propostas de intervenção para as sociedades ? Tratase do problema da implicação ou predição possivel, a partir de evidências construídas no plano coletivo (portanto em um plano particular) para os demais planos ou dimensões da realidade, quais sejam, o plano singular dos indivíduos enquanto unidades isoladas e o plano universal das sociedades enquanto totalidades complexas (Barreto \& Alves, 1994; Taubes, 1995).

Finalmente, cabe assinalar o progressivo direcionamento das práticas de prevenção e controle para o chamado enfoque de risco, isto é, para a focalização em segmentos populacionais identificados como portadores de maiores riscos em relação a certos problemas, e o conseqüente abandono da tradição anterior de intervenções massivas nas quais todos eram igualmente afetados pelas medidas adotadas. Há aqui uma forte contradição entre os princípios de autonomia e liberdade e sua possibilidade concreta de realização, e um enorme descompromisso do Estado face aos grupos marginalizados e excluídos da sociedade. Constrói-se uma falsa equação entre "autoritarismo sanitário", de um lado, e "mercantilismo neo-liberal", de outro. Será possivel conciliar, no plano das atividades de saúde, as necessidades de afirmação de indivíduos conscientes que intencionalmente fazem sua história e as responsabilidades do
Estado em modificar as condiçōes de existência, tornando-as mais saudáveis para todos?

\section{Tendências de Desenvolvimento da Epidemiologia}

Para avaliar as tendências de desenvolvimento da área é preciso considerar, separadamente, aquilo que poderíamos denominar de epidemiologia hegemônica e epidemiologia contrahegemônica.

Em relação à epidemiologia hegemônica a primeira questão que se apresenta é até que ponto existe, nesse grupo de praticantes, a percepção da crise de paradigma. Aparentemente, pelo volume das publicações especializadas e da divulgação nos meios de comunicação de massa, a epidemiologia hegemônica vai muito bem, obrigada! Entretanto, os primeiros sinais da crise se fazem sentir entre alguns acadêmicos americanos, no que diz respeito à produção teórica, e nas associações médicas, naquilo que se refere à "falência" da estrutura de Saúde Pública nos países centrais, principalmente nos Estados Unidos (Tauber, 1995; Federation of American Scientists, 1995).

Há sinais evidentes do esgotamento do modelo de risco, tanto no que se refere às suas insuficiências teóricas quanto à sua relativa ineficiência prática, isto é, à incapacidade de produzir um discurso coerente e facilmente assimilável pelas pessoas, em termos de prevenção das doenças.

As tendências que se esboçam na epidemiologia hegemônica poderiam ser caracterizadas como tentativas de maior "tecnificação" e formalização do campo, expressas na redução dos desenhos de investigação ao ensaio clínico controlado, como se observa na vertente da epidemiologia clínica; no aprofundamento das investigações de marcadores genéticos tomados como "causa única" na produção das doenças; e na redução de toda explicação à modelagem matemática. 
A epidemiologia contrahegemônica, por sua vez, vivencia uma certa dificuldade de se estabelecer como alternativa à crise paradigmática. As tendências de superação da crise apontam em duas direções distintas. De um lado, há uma tentativa de, permanecendo no mesmo sistema de lógica formal, revalorizar as abordagens ecológicas como sucedâneo para a complexidade e para a exigência de uma compreensão da totalidade. Do mesmo modo, busca-se uma leitura das contribuições da "epidemiologia molecular", em que as interaçōes genótipo/fenótipo mediadas pela cultura "senso lato" substituem a visão simplista de determinismo causal (Susser, 1995).

Por outro lado, há todo o desenvolvimento promovido pela epidemiologia social latino-americana buscando incorporar a compreensão da gênese do processo saúde-doença na perspectiva da determinação social, superando assim a carência de uma teoria no âmbito da epidemiologia hegemônica $\mathrm{e}$, ao mesmo tempo, reafirmando o compromisso histórico da disciplina com a denúncia e a superação das desigualdades sociais. Como afirma Breilh (1994), diante do "fogo cruzado" em que a Epidemiologia se encontra, resta aos praticantes optar entre três possíveis caminhos: assumir a vitória da posição neo-liberal e acomodar-se ao trabalho funcional e apolítico da corrente hegemônica; impulsionar a "terceira via" da social-democracia reformista; ou retomar o espírito crítico e a prática da transformação socialista.

\section{Referências bibliográficas}

ABRASCO (1986) - I Reunião Nacional sobre Ensino e Pesquisa em Epidemiologia. Estudos de Sauide Coletiva 4:91-108.

ABRASCO (1990) - Epidemiologia e Desigualdade social: os desafios do final do século. Anais do I Congresso Brasileiro de Epidemiologia, Campinas.

ABRASCO (1992) - Qualidade de vida: compromisso histórico da Epidemiologia. Anais do II Con-
A história das idéias e práticas na Saúde coletiva e, em particular na Epidemiologia, tem sido marcada pela oposição permanente entre compromissos reformistas e revolucionários. Daí o dilema vivido permanentemente pelos epidemiologistas, entre atuar para minimizar, no plano dos fenômenos (vale dizer, do consumo), as condições decorrentes da formação social, ou desvelar essas contradições e trabalhar na direção de sua superação, ainda que no âmbito restrito da saúde e da doença.

A primeira e mais fundamental questão ética que se impooe ao pesquisador é a aceitação ou o rechaço das condições concretas nas quais sua atividade de pesquisa terá que se desenvolver, isto é, a consideração do senticlo segundo o qual seu próprio trabalho participará da construção e/ou transformação das regras de estruturação e funcionamento da sociedade. Caberia talvez lembrar a máxima atribuída a Protágoras - "o homem é a medida de todas as coisas" - ou seja, as coisas só adquirem valor do ponto de vista da vida humana; todo conhecimento só adquire valor a partir da perspectiva de sua aplicabilidade e utilidade prática para o homem.

Importa recuperar para a prática científica a dimensão ética, em seu sentido mais amplo, de compromisso dos cientistas com a realização da essência do homem enquanto ser genérico, não apenas naquilo que a atividade humana tenha de potencialidade para o controle da Natureza, mas, principalmente, naquilo que ela possa ter de busca da felicidade.

gresso Brasileiro de Epidemiologia. Belo Horizonte: COOPMED/ABRASCO.

ABRASCO (1995) - A epidemiologia na busca da equidade em saúde. Resumos do III Congresso Brasileiro de Epidemiologia, Salvador.

ALMEIDA-FILHO, N. (1992) - La pratica teorica de la epidemiologia social en America Latina. Salud y Cambio 10:25-31. 
ALMEIDA-FILHO, N. (1994) - Apuntes para la reconstrución teórica de la epidemiología. Conferência apresentada no III Congresso Panamericano de Epidemiologia, Córdoba, Argentina.

ALMEIDA-FILHO, N. (1995) - Les défis de l'épidémiologie sociale latino-américaine Ruptures, Revue transdiciplinaire en santé 2(1):8-16.

AMANN, K. \& KNORR-CETINA, K. (1990) - The fixation of (visual) evidence. In: Lynch M., \& Woolgar, S. (orgs.) - Representation in Scientific Practice. Cambridge, Massachussets: Cambridge MIT Press.

AYRES, J.R.C.M. (1992) - Elementos históricos e filosóficos para a crítica da epidemiologia. Anais do II Congresso Brasileiro de Epidemiologia, Belo Horizonte.

AYRES, J.R.C.M. (1994) - Interpretaçào histórica e transformação científica: a tarefa hermenêutica de uma teoria crítica da epidemiologia. Revista de Saúde Puiblica 28(4):311-319.

AYRES, J.R.C.M: (1995) - Necessidade, objetividade e risco: ensaio para uma propedêutica epistemológica. Conferência apresentada no III Congresso Brasileiro de Epidemiologia, Salvador.

BARATA, R.B. (1990) - Avaliaçào dos Sistemas Locais de Sańde: a perspectiva da epidemiologia. Washington: OPS.

BARATA, R.B. (1993) - Reorientação das práticas de vigilância epidemiológica. Anais do Seminário Nacional de Vigilância Epidemiológica. Brasilia. CENEPI/FNS

BARRETO, M.L. \& ALVES, P.C. (1994) - O coletivo versus $\mathrm{O}$ individual na epidemiologia: contradição ou síntese? In: Lima \& Costa, M.F.F. \& Sousa, R.P. (orgs.) - Qualidade de vida: compromisso bistórico da epidemiologia. Belo Horizonte: COOPMED/ABRASCO.

BREILH, J. (1994) - Dialéctica de lo colectivo en epidemiologia. In: Lima \& Costa, M.F.F. \& Sousa, R.P. (orgs.) - Qualidade de vida: compromisso bistórico da epidemiologia. Belo Horizonte: COOPMED/ ABRASCO.

CASTELLANOS, P.L. (1990) - Avances metodológicos en epidemiologia. Anais do I Congresso Brasileiro de Epidemiologia, Campinas.

CASTIEL, L.D. (1995) - Ondas, cadeias, teias, redes...buscando metáforas para uma epidemiologia mestiça. Trabalho apresentado em Mesa-redonda no
III Congresso Brasileiro de Epidemiologia, Salvador. CORISCO (1993) - Teoria crítica da epidemiologia. Oficina de Trabalho UFBA/OPS Salvador, mimeo.

CZERESNIA, D. (1993) - Construção científica e inovação teórica: um desafio para a epidemiologia. Physis 3(1):77-90.

FEDERATION OF AMERICAN SCIENTISTS (1995)

- Global Program to Monitor Emerging Diseases. Washington, mimeo.

KUHN, T. (1989) - A estrutura das revoluçōes científicas. São Paulo: Editora Perspectiva.

MENDES-GONÇALVES, R.B. (1990) - Contribuição à discussão sobre as relaçōes entre teoria, objeto e método. Anais do I Congresso Brasileiro de Epidemiologia, Campinas.

MENDES-GONÇALVES, R.B. (s/d) - Práticas de saúde: processos de trabalho e necessidades, mimeo (texto inédito)

MORGENSTERN, H. (1982) - Uses of Ecologic Analysis in Epidemiologic Research. American Journal of Public Health 72(12):1336-44.

PAIM, J. S. (1992) - La salud colectiva y los desafios de la practica. Publicación Cientifica 540. Washington: OPS

SAMAJA, J. (1992) - Fundamentos epistemológicos de las profesiones de la salud. Taller OPS, Caracas, mimeo.

SUSSER, M. (1995) - Choosing a Future for Epidemiology Ecologism in a Global Era. Trabalho apresentado no III Congresso Brasileiro de Epidemiologia, Salvador.

TAUBES, G. (1995) - Epidemiology Faces its Limits. Science 269:164-169.

VON KORFF, M.; KOEPSELL, T.; CURRY, S. \& DIERH, P. (1992) - Multi-level Analysis in Epidemiologic Research on Health Behaviors and Outcomes. American Journal of Epidemiology 135(10):1077-82.

WING, S. (1993) - Concepts in Modern Epidemiology: Population, Risk, Dose response and Confounding. Teoria crítica em epidemiologia - Oficina de Trabalho, UFBA/OPS, Salvador, mimeo.

WING, S. (1993) - Limits of Epidemiology: the Case of Radiation and Health. Teoria crítica em epidemiologia - Oficina de Trabalho, UFBA/ OPS, Salvador, mimeo. 